\title{
Effects of ageing on mechanical durability of round clinched steel/aluminium joints
}

\author{
Luigi Calabrese ${ }^{1}$, Edoardo Proverbio $^{1}$, Giovanna Galtieri $^{* *}$ and Chiara Borsellino²
}

\begin{abstract}
Background: The clinching is one of the most common metal joining processes in the manufacturing of metal plate based products (similar or dissimilar, pre-coated or galvanized), especially when the assembly without adding major joining elements is required. When the clinched joints work in an aggressive environment, particular attention would be placed on the electrochemical stability and corrosion resistance of the metal constituents (Mizukoshi and Okada 1997). In joining design, an appropriate material selection reduces the electrochemical potential differences and prevents significant galvanic currents (Kruger and Mandel 2011; Calabrese et al. 2014). The durability of the metal joints could be heavily influenced in a corrosive environment, whereas the less noble material will tend to increase its corrosion rate; instead the more noble one will reduce its electrochemical dissolution (He et al. 2008; Bardal 2004). Accelerated ageing tests (i.e. salt fog test) were carried out to evaluate the durability of the joints in highly aggressive environments (Calabrese et al. 2013; LeBozec et al. 2012). Although the durability for a long time of the clinched joint in a corrosion environment is a known problem, few works focus the attention on the relationship between durability of joints and electrochemical behaviour of the metal constituents. The aim of the present work is to evaluate the durability at long ageing time in salt spray test (according to ASTM B117) of carbon steel/aluminium alloy joints, obtained by clinching.

Methods: The investigation has been conducted on one total thickness $(2.5 \mathrm{~mm})$ of unsymmetrical joints (i.e. thickness sheets of $1.5 \mathrm{~mm}$ and $1 \mathrm{~mm}$ ) to inquire about the effect of corrosion on the two different unsymmetrical configurations (St1.5/Al1 and St1/Al.5). The joint resistance has been determined, by means of shear tests of single-lap joints in according to ISO/CD 12996. The samples were exposed to critical environmental conditions following the ASTM B 117 standard. To inquire the damage evolution of the samples, 0, 1, 2,3, 5, 7, 10 and 15 weeks of ageing time have been chosen. Seven samples for each combination and for each ageing time were realized. A Design of Experiment has been performed, followed by the ANOVA of the results to analyse the influence of the two factors, thickness combinations and ageing time, on the mechanical properties of the joints.

Results: The two sets of joints show a different behaviour at increasing ageing time: the St1.5/Al1 batch shows a constant decay of the load values, instead the St1/Al1.5 set maintains acceptable values of resistance for several weeks of ageing, at tenth week the mechanical stability is strongly impaired. In the latter case the presence of the thin oxide layer at the overlapping interface, which behaves as an adhesive interlayer, and the larger thickness of the aluminium plate improve the resistance of the St1/Al1.5 joints. Statistical analysis confirms that the two thickness combinations and ageing time are the significant factors. At zero weeks, neglecting the effect of ageing, the maximum load values of all samples belong to the same population. This means that the resistance of the clinched joints is the same regardless the combination of thicknesses, but by considering both the ageing and thickness, the analysis of variance shows that both thickness and weeks are significant parameters distinguishing two different populations in the distribution of loads.

(Continued on next page)
\end{abstract}

\footnotetext{
* Correspondence: ggaltieri@unime.it

1 Department of Electrical Engineering, Chemistry and Industrial Engineering,

University of Messina, Contrada di Dio, 98166 Messina, Italy

Full list of author information is available at the end of the article
} 
(Continued from previous page)

Conclusions: The experimental results evidenced that the corrosion degradation phenomena influence significantly both the performance and the failure of the joints. This is also confirmed by statistical analysis according to which the two thickness combinations and ageing time are the significant factors.

Keywords: Clinching; Corrosion; Durability; Salt spray environment test; Single-lap shear test

\section{Background}

The clinching is one of the most common metal joining processes in the manufacturing of metal plate-based products, especially when the assembly without adding major joining elements is required. This joining technique is indicated for coupling, similar or dissimilar, pre-coated or galvanized, material sheets up to a total thickness of $3 \mathrm{~mm}$ (Nong et al. 2003; Mucha 2007; Mucha et al. 2011; Di Lorenzo and Landolfo 2004; Varis 2003; Borsellino et al. 2004).

The clinching process joins two sheets, placed between a punch and die, by a localized cold forming. A button is formed on the underside and provides an interlock between the plates. The interlocking friction joint can be realized between two or more overlapped layers of material.

The clinching is a technological solution that does not affect the performances or introduce physical discontinuity between the constituent materials; in fact, it does not induce any thermal stresses into the workpiece. The process causes no heat, no splashes, no flashes or harmful light.

The mechanical stability of clinched junctions is influenced by several factors such as the correct selection of process parameters or the geometry of assembled materials (Varis and Lepisto 2003; Lee et al. 2010; Oudjene and Ben-Ayed 2008). Saberi et al. (Saberi et al. 2008) have analysed different surface conditions (blank, electro galvanized, corrosion protection primer coated) to assess their influence on the geometry and mechanical strength of the clinched joint, comparing experimental results with the numerical one by finite element analysis.

When the clinched joints work in an aggressive environment, particular attention would be placed on the electrochemical stability and corrosion resistance of the metal constituents (Mizukoshi and Okada 1997). In joining design, an appropriate material selection reduces the electrochemical potential differences and prevents significant galvanic currents (Kruger and Mandel 2011; Calabrese et al. 2014). The durability of the metal joints could be heavily influenced in a corrosive environment, whereas the less noble material will tend to increase its corrosion rate; instead, the nobler one will reduce its electrochemical dissolution (He et al. 2008; Bardal 2004).

The presence of surface irregularities or crevices leads to crevice corrosion attack, due to electrolyte accumulation in the interstices. Furthermore, another aspect that influences the durability in aggressive environmental conditions is the presence of internal stresses induced by cold forming procedure. The activation and propagation of local cracks due to stress corrosion cracking (SCC) weaken furthermore the clinched joint at long ageing times (Li et al. 2010; Winzer et al. 2005).

The aluminium sheet in the clinched joint will be subject to oxide film formation that will act as protective barrier between the metal and the surrounding medium if the $\mathrm{pH}$ value of the environment is in the range of about 4 to 8 . Below and above these values, the acid and alkaline dissolution of $\mathrm{Al}$ plate yields $\mathrm{Al}^{3+}$ and $\mathrm{AlO}^{-2}$ ions, respectively. Consequently, the oxidised metal leads to breakage due to the thinning induced by electrochemical dissolution (UK Aluminium Industry Fact Sheet 2 2012).

The overlapping area affected by corrosion phenomena, progressively increases during ageing time, considering that the electrolyte gradually penetrates towards the centre of the overlap. After a sufficient accumulation of the corrosion products, a loss of direct contact between the metals occurs and this could compromise the interlocking of the joint.

Accelerated ageing tests (i.e. salt fog test) were carried out to evaluate the durability of the joints in highly aggressive environments (Calabrese et al. 2013; LeBozec et al. 2012). Moroni et al. (Moroni et al. 2010) have shown the influence of thermal cyclic ageing on the performances of hybrid adhesive-mechanical joints, whereas ageing influences slightly the performances of hybrid joints. Although the durability for a long time of the clinched joint in a corrosion environment is a known problem, few works focus the attention on the relationship between durability of joints and electrochemical behaviour of the metal constituents.

In particular, (LeBozec et al. 2008) have shown that the corrosion resistance of basic materials (e.g. steel or zinccoated steel) and consequently, their durability performances are highly dependent on the kind of used acceleratedcorrosion test. Consequently, the uncertainty about the degradation mechanisms induced by corrosion of the clinched joint is still relevant. At the same time, the variety of the joint type in the automotive design, as well as the assembly procedure, make any theoretical approach very difficult.

In this context, the present work has the purpose to evaluate the durability at long ageing time in salt spray test (according to ASTM B117) of carbon steel/aluminium alloy joints, obtained by clinching. The joint resistance has been determined by means of single-lap shear tests after each ageing cycle. A design of experiment has been performed, followed by the analysis of variance 
(ANOVA) of the results to evaluate the effects of both 'substrate thickness' and 'accelerate ageing'.

Two combinations of aluminium alloy/carbon steel thicknesses were investigated; seven samples for each combination and for each ageing time were realized.

The observed results can be helpful in engineering design to optimize the joining process (properties, materials configuration, thickness, etc....), taking into account the durability in aggressive environmental condition of the joints during its service life.

\section{Methods}

\section{Materials and manufacturing}

The investigation has been carried out on unsymmetrical single-lap joints with a total thickness of $2.5 \mathrm{~mm}$ to inquire about the effect of corrosion on the sheet configuration. The employed materials, their thickness, their chemical and mechanical properties are reported in Table 1.

Clinched joints were realized with the two different combinations of thickness: St1.5/Al1 [mm] and St1/Al1.5 [mm]; where St stands for the steel sheet at top and $\mathrm{Al}$ for the aluminium-alloy, this latter is always placed at the bottom during the clinching procedure. The geometry of clinched single-lap joint is shown in Figure 1a. The aluminium plate, more ductile than the steel one, deforms filling the cavity of the die and allowing the plastic deformation of the St plate on which the punch acts. The correct pressure affects the efficiency of the joint. Figure $1 \mathrm{~b}$ shows the cross sections at 0 weeks respectively for the St1.5/Al1 and St1/Al1.5.

To inquire the damage evolution of the samples, $0,1,2$, $3,5,7,10$ and 15 weeks of ageing time have been chosen. For each ageing time, seven samples of each configuration were prepared.

\section{Clinching process}

The joints were realized by an electro-hydraulic riveting system. The duration of the process was about $2 \mathrm{~s}$. The equipment (Textron Fastening System) was supplied by a hydraulic motor $(230 \mathrm{~V}, 50$ to $60 \mathrm{~Hz})$ with an electrohydraulic valve necessary to vary the pressure applied on

Table 1 Properties of the employed materials

\begin{tabular}{lll}
\hline & Material & \\
\cline { 2 - 3 } & AA6082 & $\begin{array}{l}\text { Carbon } \\
\text { steel A570 }\end{array}$ \\
\hline Geometry (thickness in $\mathrm{mm})$ & 1 to 1.5 & 1 to 1.5 \\
Chemical composition & $\mathrm{Fe}=0.5 \mathrm{Cu}=0.1 \mathrm{Si}=0.5$ & $\mathrm{C}=0.3$ \\
& $\mathrm{Mn}=0.4 \mathrm{Mg}=0.6$ to 1.2 & $\mathrm{Si}=0.25$ \\
& $\mathrm{Cr}=0.25 \mathrm{Zn}=0.2 \mathrm{Ti}=0.1$ & $\mathrm{Mn}=0.8$ \\
& $\mathrm{Al}=$ balance & $\mathrm{P}=0.04$ \\
Hardness & $\mathrm{HBN}(2.5 / 62.5 / 30)=60$ & $\mathrm{HV}=170$ \\
Yield strength $(\mathrm{MPa})$ & 224 & 590 \\
\hline
\end{tabular}

the punch. The max operation pressure was 630 bar; the maximum force was approximately $60[\mathrm{kN}]$. The die has cylindrical shape with flat bottom, and its walls are split into four sectors that expand driven by the flow of the forming material, counteracted by the action of a spring. The pressure applied to the punch allows to optimize the shape of the round button and hence its mechanical resistance. Prior to the clinched sample preparation, several attempts were done at varying the oil pressure to optimize the pressure for both configurations. The working pressure was 300 bar for both St1.5/Al1 [mm] and St1/Al1.5 [mm].

\section{Ageing treatment}

The samples were exposed to critical environmental conditions following the ASTM B 117 standard. The salt fog had a chemical composition of $5 \% \mathrm{NaCl}$ solution (pH between 6.5 and 7.2). In the climatic chamber, the samples were aged continuously with a temperature of $35^{\circ} \mathrm{C}$.

At each fixed ageing time, seven specimens of each configuration were removed and mechanically tested. Then, the samples, wished and dried, were preserved in a sealed plastic storage bag with silica gel desiccant to ensure no further corrosion during storage; moreover, the appropriate actions have been taken to avoid the introduction of other variable factors, such as the control of the environment conditions.

\section{Single-lap shear test}

Shear tests of single-lap joints were performed, according to ISO/CD 12996, by means of an Universal Testing Machine (Zwick-Roell Z250, Zwick GmbH \& Co. KG, Ulm, Germany) equipped with a $250[\mathrm{kN}]$ load cell and a cross-head rate of $1 \mathrm{~mm} / \mathrm{min}$ (displacement control test).

\section{Statistical analysis}

To analyse the influence of the two factors, thickness combinations and ageing time, on the mechanical properties of the joint, the ANOVA was performed by Minitab ${ }^{\circ}$ software (Minitab Inc., State College, PA, USA).

In the following, the two factors 'thickness' and 'ageing time' are called thickness and week, respectively; the first with two levels (level 1 for St1/Al1.5 and level 2 for St1.5/Al1), the second with eight levels ( 1 for 0 weeks, 2 for 1 week, 3 for 2 weeks, 4 for 3 weeks, 5 for 5 weeks, 6 for 7 weeks, 7 for 10 weeks and 8 for 15 weeks).

\section{Results and discussion Single-lap shear tests}

Figures 2 and 3 show the load versus displacement trends of the single-lap shear tests for two joint configurations at increasing ageing time.

The resistance of the clinched joints decreases over time due to ageing in salt spray environment. The trends, at first, show a linear increase of the load. In this 

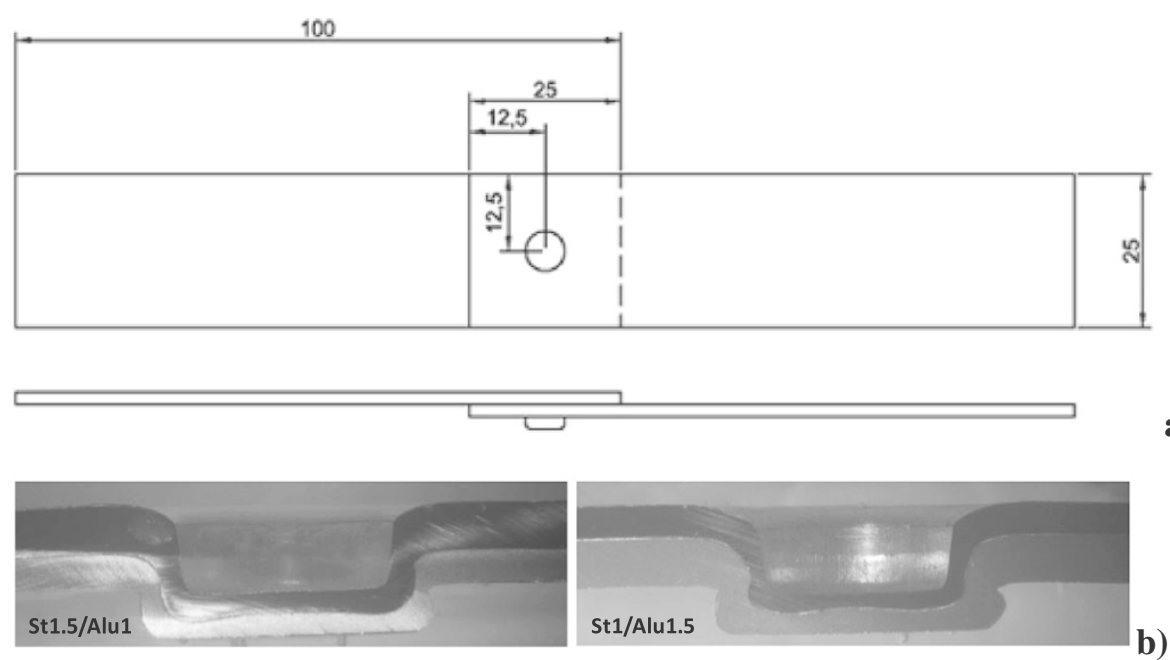

a)

Figure 1 Single-lap joint geometry $[\mathrm{mm}](\mathrm{a})$ and the cross sections of both sets (b).

phase, the joint resistance is due both to the friction resistance, resulting from the contact pressure between the aluminium and steel sheets induced by forced mechanical joining. In this linear region, the maximum slope of the load/displacement curve is reached. After this phase, the activation of damage mechanisms of the joint occurs. In particular, the samples, at low ageing time, lose gradually their stiffness. This is due to a bending effect at the edge of the sheets in the overlapping area. After that, the maximum load value is reached, and the mechanical stability of the joints is critically prejudiced. Then, the slope of the load/displacement curve becomes negative with a gradual reduction of load, thus confirming that the mechanical interlocking is compromised. The joints subsequently open as a press-stud, such rupture occurs in the most of the clinched joints (Varis 2006). The ageing treatment reduces significantly the performances of the joint giving rise premature failure mechanisms induced by localized corrosion phenomena. At long ageing times, where the joint presents an advanced state of degradation, breakages in the neck of the joint both on the aluminium side and on the steel one could occur.

The St1.5/Al1 joints show displacements smaller than those of St1/Al.5 set, breaking for unbuttoning before of the other set. This behaviour could be due to a small 'S' shape of the joints where the thick steel sheet undergoes a smaller deformation than the underlying thin aluminium sheet. This favours a reduction of interlocking force between the sheets.

With increasing ageing time in all samples, the maximum load progressively decreases and conversely, the displacement to failure increases. This is related with the electrochemical dissolution by metallic corrosion of its constituents. The aluminium sheet acts as anode and steel one as cathode. Consequently, the

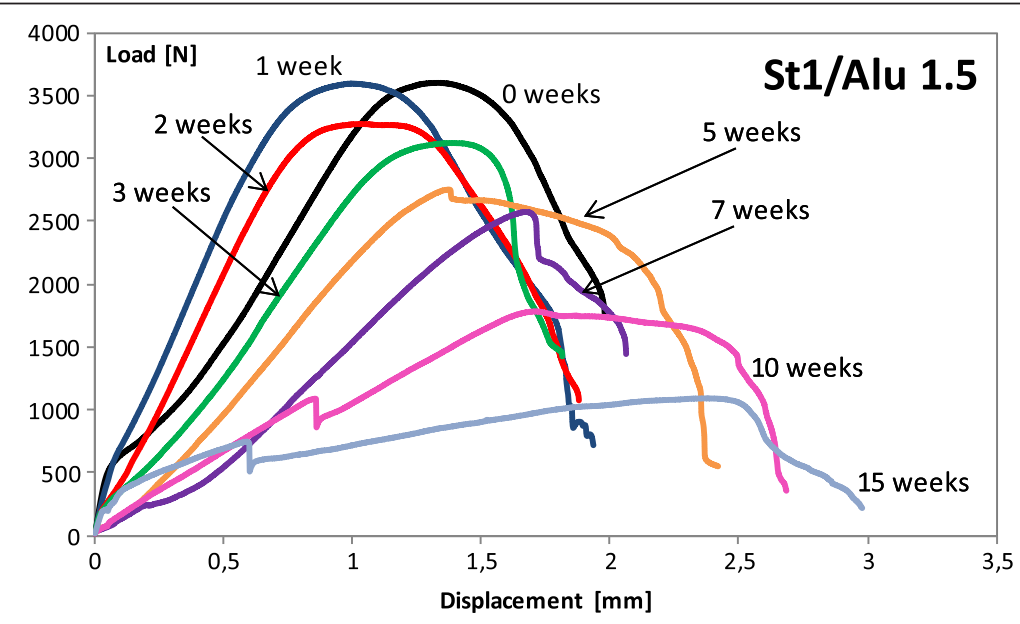

Figure 2 Load/displacement curves for St1/Al1.5 samples at increasing ageing time. 


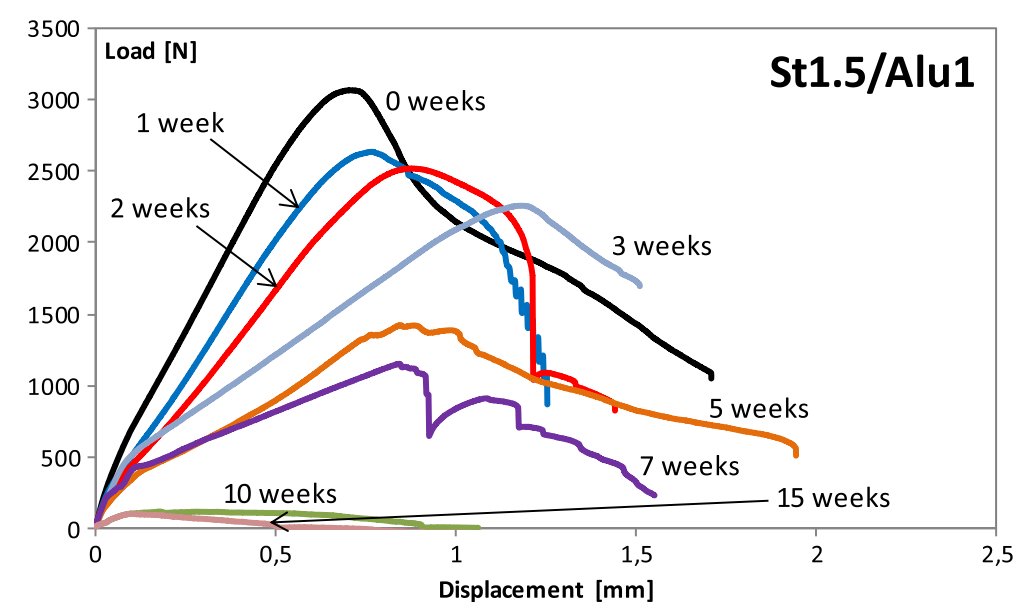

Figure 3 Load/displacement curves for St1.5/Al1 samples at increasing ageing time.

degradation phenomena will be heavy exalted on the aluminium support, due to the $\mathrm{Al} / \mathrm{Fe}$ galvanic coupling. This influences significantly the failure behaviour of the joints.

The permanence for a long time in the salt spray environment occasionally leads to premature rupture of some joints in the climatic chamber, due to heavy galvanic corrosion on the aluminium sheet.

The trend of the mean values of the maximum load, obtained from the shear tests, at varying exposure time in salt spray environment was recorded and it is reported in Figure 4.

At 0 weeks, both configurations of the joints, in spite of an uneven geometry, show a good mechanical resistance with still acceptable values up to first 2 weeks.

The two sets of joints show a different behaviour at increasing ageing time: the St1.5/Al1 batch shows a constant decay of the load values, instead, the St1/Al1.5 set maintains acceptable values of resistance for several weeks of ageing and at tenth week, the mechanical stability is strongly impaired. In the latter case, the presence of the thin oxide layer at the overlapping interface, which behaves as an adhesive interlayer, and the larger thickness of the aluminium plate improve the resistance of the St1/Al1.5 joints.
In aggressive environmental condition, such as the salt spray fog, the aluminium sheet undergoes a relevant degradation for galvanic corrosion. Oxides and hydroxides of $\mathrm{Al}$ (i.e. $\mathrm{Al}_{2} \mathrm{O}_{3}, \mathrm{Al}(\mathrm{OH})_{3}$ ), formed at long ageing times (noticeable by their typical white coloration), induce an increase of volume in the joining area, resulting in bulging or pillowing of the overlapped sheets, as shown in Figure 5.

Thus, the presence of $\mathrm{Al}$ corrosion products influences the interlocking of the clinched button. At first, when the oxide layer is thin, an improvement of the interlocking can be observed. Indeed, after few weeks, the failure load, that should decrease due to the galvanic corrosion, instead is quite similar to the unaged samples. When the layer of oxides becomes thick, its brittle behaviour influences significantly the failure. In fact, for medium and long ageing times, a progressive reduction of the joint strength is observed. Furthermore, due to the galvanic corrosion, the aluminium sheet undergoes a progressive thinning, especially in the overlapped area. This effect is strongly evident at long ageing times (especially for samples with thinner aluminium sheet, as shown in Figure 6), because the aluminium dissolution affects a large area of the joint, inducing a critical damage in correspondence of the clinched area.

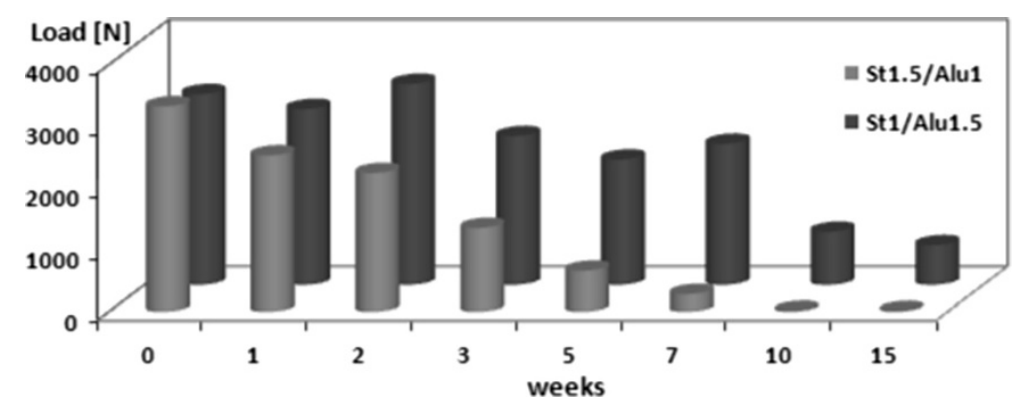

Figure 4 Mean values of max load (from shear test) at varying exposure time. 

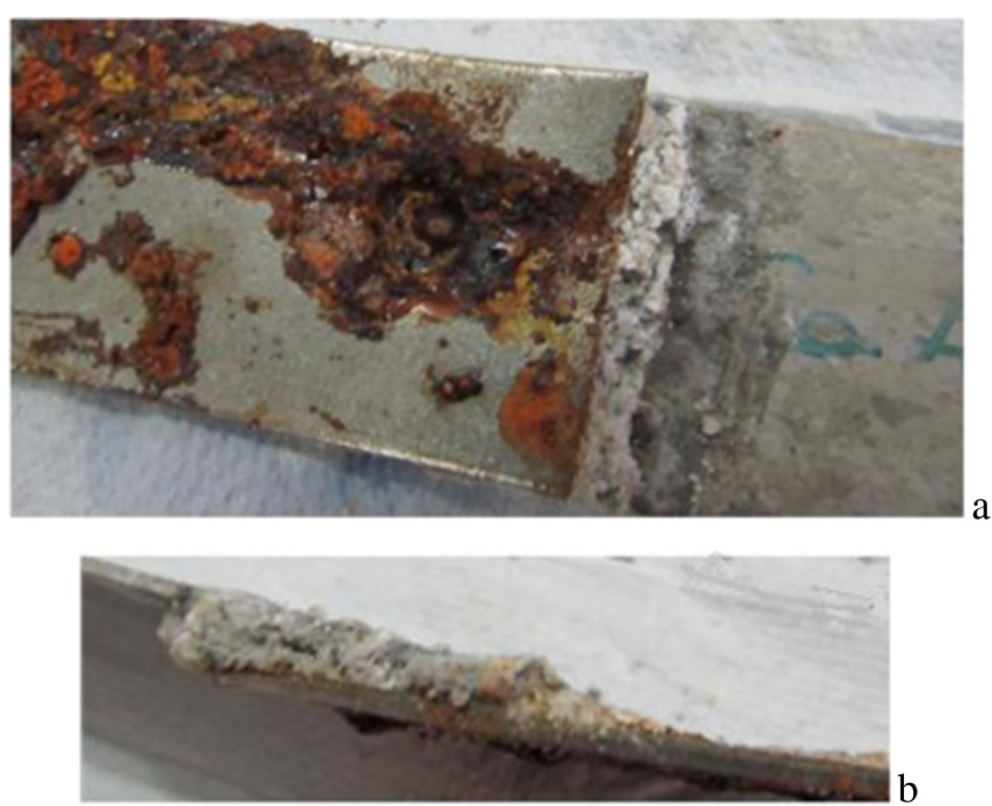

Figure 5 Al oxide layer in St1/Al1.5 (a) and in St1.5/Al1 (b).

Consequently, a significant drop of performances takes place, compromising definitely the mechanical stability of the joint.

From Figure 4, we can distinguish three stages depending on the ageing time to evaluate the performance decay for both kinds of joints due to the salt spray effects:

- At first, a stability region is present. The samples exhibit a slight reduction of their performances. This region is evident especially for the St1/Al1.5.

- As for medium ageing times, a significant progressive decay of the mechanical resistance of the joints takes place.

- Finally, for long ageing times, very low resistance values occur. For the St1.5/Al1 batch, this region lies at 5 weeks, whereas for the St1/Al1.5 batch, this phenomenon is evident at about 10 weeks.

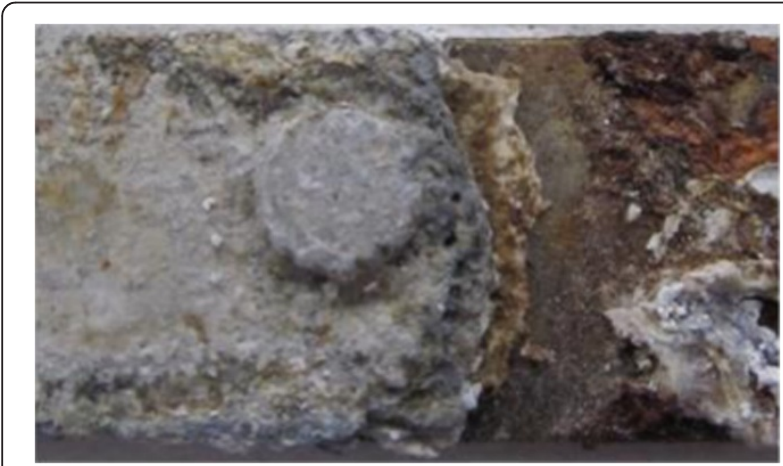

Figure 6 Progressive Al thinning in a St1/Al1.5 joint at 10 weeks.
With the aim to inquire about the reliability of the joints, the load values have been ordered from the maximum to the minimum at each ageing time and the series of such values have been plotted with the ageing time; their trend is reported in Figures 7 and 8, for the St1.5/ Al1 and St1/Al1.5, respectively.

The higher curves are related to samples with low manufacturing defects, characterized by better performance and higher mechanical stability to corrosion degradation. Instead, the lower curves are related at samples sensible to corrosion. At long ageing time, some curves disappear from the graph because some samples have reached the failure in the climatic chamber (without tensile test) due to metal dissolution.

It is worth noticing the different trends of the two series of joints: in Figure 7, the joints still maintain an



Figure 7 Ordered load at each exposure time - St1.5/Al1. 


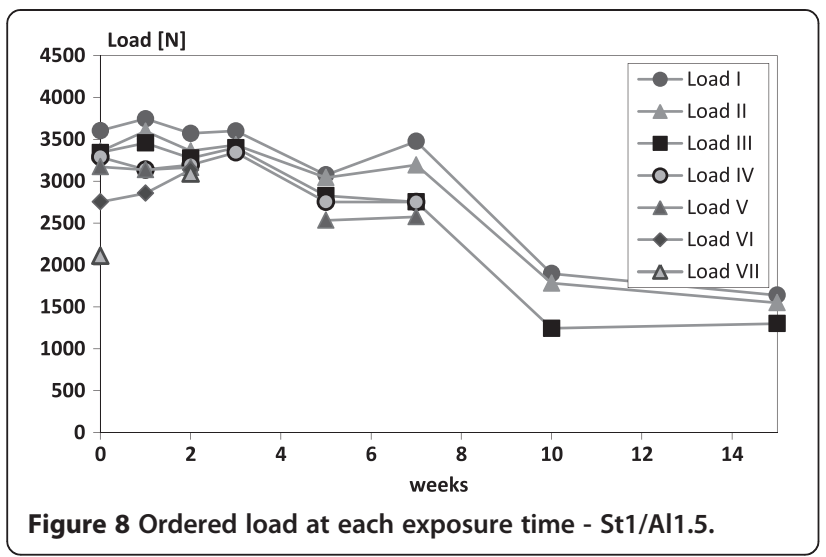

acceptable resistance (i.e. 30\% less) and all the samples are even intact for the first 2 weeks; in the climatic chamber, the number of broken samples increases at longer ageing time and in fact, at 10 and 15 weeks, only one sample, among seven, is evaluable for the experimental test. In Figure 8, the samples are all intact and in the same range of load values until 3 weeks, then the number of broken samples increases at increasing ageing, but, in this case, the unbroken samples show acceptable resistance (i.e. $30 \%$ less) until 7 weeks.

\section{Statistical analysis}

\section{Data set at '0 weeks'}

The ANOVA analysis allows evaluating the effect of each factor and their interaction. In a first step of analysis, the factor weeks have been neglected and the one-way ANOVA has been applied on the data at 0 weeks to investigate whether the thickness is a significant factor. Because the obtained $p$ value is equal to 0.37 , it is possible to affirm that the load values belong to the same population and it is not possible to distinguish between the two set of samples (St1.5/al1 and St1/Al1.5). Both the Tukey 95\% and Fisher 95\% tests confirm such statement finding that the distribution of 'difference of the loads' has the mean value near to zero. Figure 9 evidences that the two distributions of failure loads of the two sample batches lie in two overlapping ranges.

\section{Data set at all ageing time}

Since the data are balanced (seven replicates for each case study) and the factors are fixed (St1.5/Al1 and St1/ Al1.5 are respectively thickness 1 and 2 - ageing times 0 , $1,2,3,5,7,10$ and 15 are respectively weeks $1,2,3,4,5$, 6, 7 and 8), the two-way analysis of variance is applied (see Figure 10).

This analysis allows to evaluate both the effect of each factor and their interaction. Both thickness and weeks are significant factors because their $p$ value is equal to

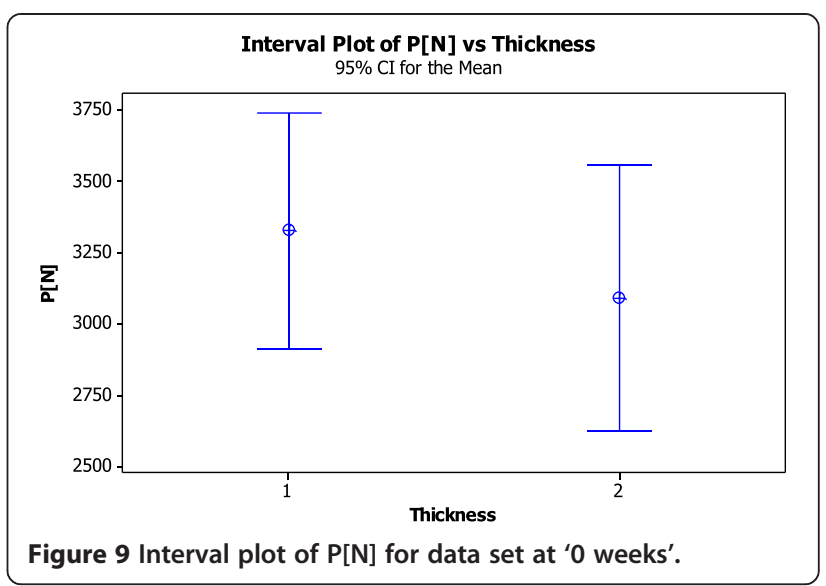

zero, and there is no interaction because $p=0.073$ (no interaction if $p>0.05$ ).

Figure 10 shows that the interaction is negligible between thickness and weeks, the data fall inside the range of confidence, except for some data being on the borderline. The main effect of the two parameters confirms that thickness and weeks are statistical significant parameters (data lying outside the range of confidence).

Moreover, both the Tukey 95\% and Fisher 95\% tests on the thickness confirm that the load values belong to different populations. In fact, the distribution of 'difference of the loads' has strictly the mean greater than zero. Since the interaction between thickness and weeks is negligible, it is possible to analyse separately the two data sets (respectively, thickness 1 and 2).

\section{Data set 'thickness 1 '}

The ANOVA performed on the data thickness 1 (St1.5/ Al1) confirmed the effect of the week factor, showing some outliers (some loads are 'zero' - indicating broken samples inside the chamber at weeks 2 and 3 - i.e. ageing time $=1$ to 2 weeks).

By performing both the Tukey 95\% and Fisher 95\% tests simultaneous confidence intervals that realize all pairwise comparisons among levels of weeks, with individual confidence level $=99.73 \%$, Figure 11 is obtained, where the load values at 'weeks $=1$ subtracted from all the others' $(\Delta$ weeks) are reported against the difference of their loads $P(\Delta P)$.

With such analysis is possible to clearly identify whether a mean load is significantly different from the others at different ageing times. This confirms a first stage (identified as pre-ageing cluster) where a slight and constant decay of the load is attained, a second one (transition cluster) where a deep decay (with respect to the initial load value) is attained and a third one (postageing cluster) where a complete loss of load carrying capability is found. 


\section{Two-Way ANOM for P[N ] by Thickness, Weeks \\ Alpha $=0.05$}
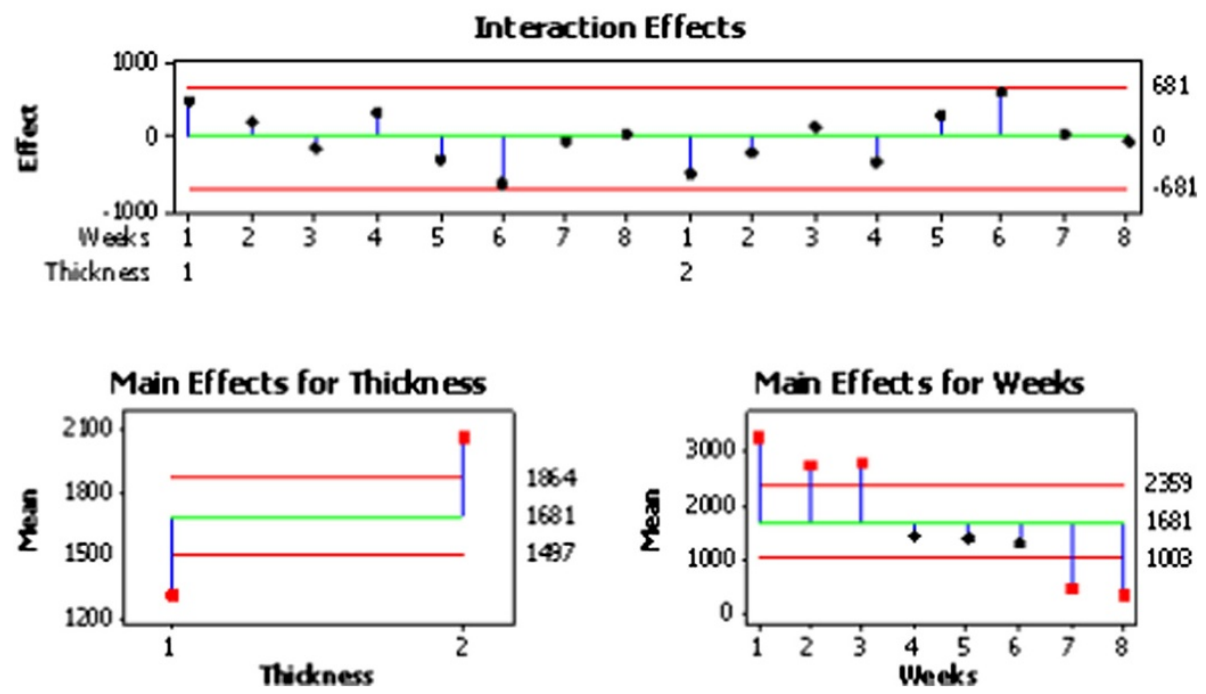

Figure 10 Two-way ANOM for the load by thickness and weeks.

\section{Data set 'thickness $2^{\prime}$}

The ANOVA performed on the thickness 2 (St1/Al1.5) has confirmed the effect of the factor weeks.

By performing both the Tukey 95\% and Fisher 95\% tests simultaneous confidence intervals, Figure 12 is obtained, where the load values at 'weeks $=1$ subtracted from all the others' ( $\Delta$ weeks) are reported against the difference of their loads $P(\Delta P)$.

Such analysis evidences the three clusters related with the damage evolution, as before. In this case, different amplitudes from the previous case are obtained, where the transition cluster is delayed of 4 weeks of real time of ageing, i.e. from week 4 for thickness 1 to week 6 for thickness 2 .

\section{Timeline of corrosion evolution clusters}

The St1.5/Al1 batch shows a constant decay of the load, identified as pre-ageing cluster, due to the formation of oxides. When the thickness of the oxide layer increases, a deep decay arises after three ageing weeks, defined as transition cluster. Finally, the joints arise a complete loss of load carrying capability at the tenth weeks, identified as post-ageing cluster, where the thinning and dissolution of the aluminium substrate for galvanic corrosion is more evident. Instead, the St1/Al1.5 batch has a constant trend of load values up to 2 weeks in spite of corrosion. The transition phase occurs after seven ageing weeks. The mechanical stability of the joints is completely impaired to 15 weeks of ageing (post-ageing

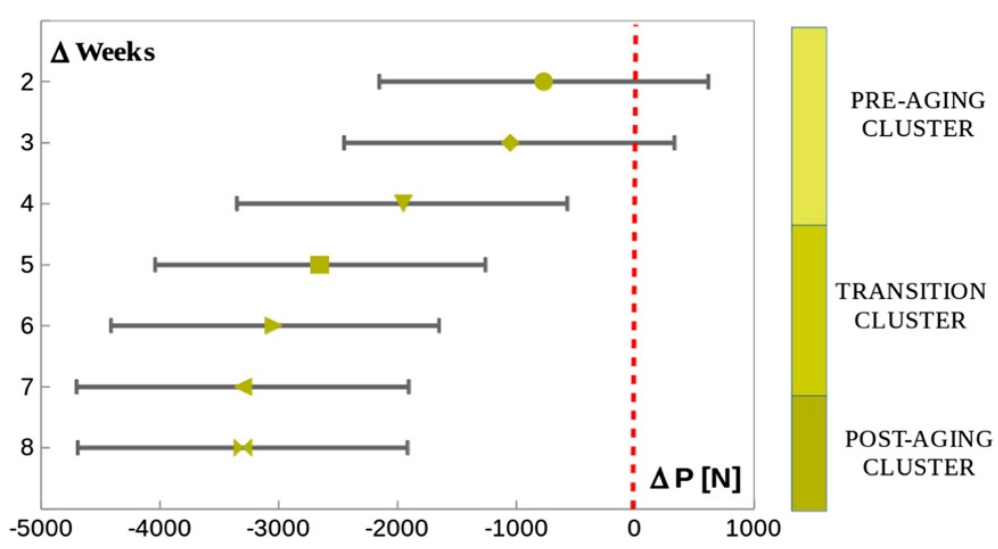

Figure 11 Pairwise comparisons among levels of weeks and respective loads - thickness 1. 


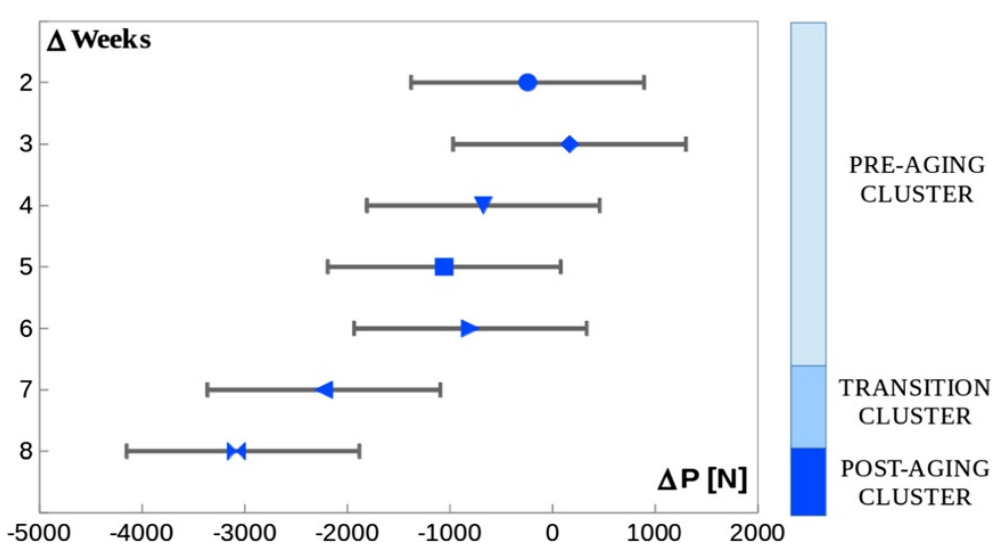

Figure 12 Pairwise comparisons among levels of weeks and respective loads - thickness 2.

cluster), when the effect of the oxides predominates compared with the interlocking.

Figure 13 shows the evolution of damage on the aluminium button for two sample sets, highlighting the effects of corrosion on the clinched joints, (e.g. the increase of oxide volume in the joining area compromises the interlocking and the thinning by dissolution of aluminium for long ageing time).

The set St1.5/Al1 undergoes a visible effect of galvanic corrosion from the third week where the erosion and thinning of the $\mathrm{Al}$ sheet was observed (see the lower central image in Figure 13) up to the full corrosion at the tenth week (see the lower right image in the same figure). At this stage, the dissolution of aluminium plate is dramatically advanced, reaching the button of the joint.

Differently, the set St1/Al1.5 (thickness 2) has shown significant effects of galvanic corrosion from the seventh week (see the upper central image in Figure 13, with formation of aluminium corrosion products mainly in the interstices of the overlapping area). Only at the 15th week, the corrosion, present in the joint overlapping area, is significant. As seen in the upper right image in the same figure, the progressive dissolution of $\mathrm{Al}$ is evident in the lower sheet edges.

\section{Conclusions}

The durability at long ageing time in salt spray test of carbon steel/aluminium alloy joints, obtained by clinching, has been evaluated. The experimental results evidenced that the corrosion degradation phenomena influence significantly both the performance and the failure of the joints; also, the joint configuration is a significant factor for the corrosion effect.

In particular, it is possible to draw out the following considerations:

- In the salt spray, the aluminium sheet undergoes a noticeable degradation for galvanic corrosion. The presence of aluminium corrosion products

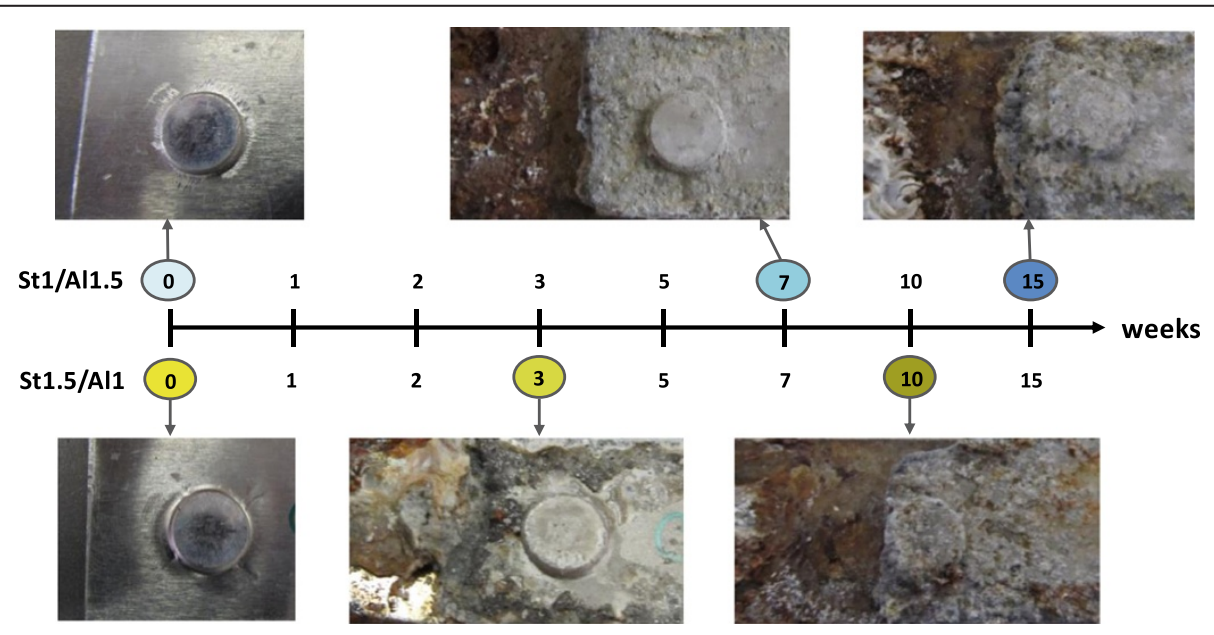

Figure 13 Evolution of damage on aluminium button at increasing ageing time. 
influences the interlocking. When the oxide layer is thin, an unexpected improvement of the interlocking was observed in which the failure load is quite similar than the unaged samples. When the layer of oxides becomes thicker, the aluminium substrate is subjected to a progressive thinning and the brittle behaviour of the oxide influences significantly the failure. This behaviour is more evident in the samples in which the metallic plate with lower electrochemical potential is thinner.

- Statistical analysis confirms that the two thickness combinations and ageing time are the significant factors. At 0 weeks, neglecting the effect of ageing, the maximum load values of all samples belong to the same population. This means that the resistance of the clinched joints is the same regardless of the combination of thicknesses, but by considering both the ageing and thickness, the analysis of variance shows that both thickness and weeks are significant parameters in distinguishing the two different populations in the distribution of loads.

\section{Competing interests}

The authors declare that they have no competing interests.

\section{Authors' contributions}

LC has participated in the durability tests studying the correlation between the corrosion phenomena and the performance of clinched joints. EP has participated in the interpretation of the metal corrosion. My contribution have concerned the joint design, the realization and discussion of the single-lap shear and aging tests, coordinating the contributions of my colleagues. CB carried out the statistical analysis explaining the fracture mechanisms. All authors read and approved the final manuscript.

\section{Author details}

${ }^{1}$ Department of Electrical Engineering, Chemistry and Industrial Engineering, University of Messina, Contrada di Dio, 98166 Messina, Italy. ${ }^{2}$ Department of Civil Engineering, Computing, Construction, Environmental and Applied Mathematics, University of Messina, Contrada di Dio, 98166 Messina, Italy.

Received: 25 April 2014 Accepted: 21 October 2014

Published online: 12 November 2014

\section{References}

Bardal, E. (2004). Corrosion and protection. Berlin: Spriger Verlab.

Borsellino, C, Buffa, G, Fratini, L, \& Ruisi, VF. (2004). Technological alternatives in the joining of lightweight aluminum structures. Seville, Spain: ISOMA - World Automation Congress 9th International Symposium on Manufacturing with Applications

Calabrese, L, Bonaccorsi, L, Proverbio, E, Di Bella, G, \& Borsellino, C. (2013). Durability on alternate immersion test of self-piercing riveting aluminium joint. Materials and Design, 46, 849-856.

Calabrese, L, Proverbio, E, Galtieri, G, \& Borsellino, C. (2014). Effect of galvanic corrosion on the durability of aluminium/steel self-piercing rivet joints. Corrosion Engineering Science and Technology. Online ISSN: 1743278214 Y0000000168.

Di Lorenzo, G, \& Landolfo, R. (2004). Shear experimental response of new connecting systems for cold-formed structures. Journal of Constructional Steel Research, 60, 561-579.

He, X, Pearson, I, \& Young, K. (2008). Self-pierce riveting for sheet materials: state of the art. Journal of Materials Processing Technology, 199(1-3), 27-36.

Kruger, L, \& Mandel, M. (2011). Electrochemical behaviour of aluminium/steel rivet joints. Corrosion Science, 53, 624-629.
LeBozec, N, Blandin, N, \& Thierry, D. (2008). Accelerated corrosion tests in the automotive industry: a comparison of the performance towards cosmetic corrosion. Materials and Corrosion, 59, $\mathrm{N}^{\circ} 11$.

LeBozec, N, LeGac, A, \& Thierry, D. (2012). Corrosion performance and mechanical properties of joined automotive materials. Materials and Corrosion, 63(5), 408-415.

Lee, C-J, Kim, J-Y, Lee, S-K, Ko, DC, \& Kim, B-M. (2010). Design of mechanical clinching tools for joining of aluminium alloy sheets. Materials and Design, 31, 1854-1861.

Li, X, Xiong, B, Zhang, Y, Wang, G, Li, Z, Zhu, B, Wang, F, \& Liu, H. (2010). Effect of ageing treatment on properties and microstructure of an Al-7.5Zn-1.3Mg1.4Cu-0.12Zr alloy. Material Science Forum, 638-642, 273-278.

Mizukoshi, H, \& Okada, H. (1997). Fatigue properties of mechanical fastening joints. Materials Science Forum, 242, 231-238.

Moroni, F, Pirondi, A, \& Kleiner, F. (2010). Experimental analysis and comparison of the strength of simple and hybrid structural joints. International Journal of Adhesion and Adhesives, 30(5), 367-379.

Mucha, J. (2007). Modern mechanical on press joinability techniques for sheet metal elements. In Proceedings of the International Conference Progressive Technologies and Materials (pp. 43-44) [in Polish].

Mucha, J, Kasak, L, \& Spisak, E. (2011). Joining the car-body sheets using clinching process with various thickness and mechanical property arrangements. Archives of Civil and Mechanical Engineering, XI, 135-148.

Nong, N, Keju, O, Yu, Z, Zhiyuan, Q, Changcheng, T, \& Feipeng, L. (2003). Research on press joining technology for automotive metallic sheets. Journal of Material Processing Technology, 137, 159-163.

Oudjene, M, \& Ben-Ayed, L. (2008). On the parametrical study of clinch joining of metallic sheets using the Taguchi method. Engineering Structures, 30, 1782-1788.

Saberi, S, Enzinger, N, Vallant, R, Cerjak, H, Hinterdorfer, J, \& Rauch, R. (2008). Influence of plastic anisotropy on the mechanical behavior of clinched joint of different coated thin steel sheets. International Journal Material Form Suppl., 1, 273-276.

UK Aluminium Industry Fact Sheet 2. (2012). Aluminium and corrosion, aluminium federation.

Varis, JP. (2003). The suitability of clinching as a joining method for high-strength structural steel. Journal of Materials Processing Technology, 132, 242-249.

Varis, J. (2006). Ensuring the integrity in clinching process. Journal of Materials Processing Technology, 174, 277-285.

Varis, J, \& Lepisto, J. (2003). A simple testing-based procedure and simulation of the clinching process using finite element analysis for establishing clinching parameters. Thin Wall Structures, 41, 691-709.

Winzer, N, Atrens, A, Song, G, Ghali, E, Dietzel, W, Kainer, KU, Hort, N, \& Blawert, C. (2005). A critical review of the stress corrosion cracking (SCC) of magnesium alloy. Advanced Engineering Materials, 7(8), 659-693.

\section{doi:10.1186/s40712-014-0023-6}

Cite this article as: Calabrese et al: Effects of ageing on mechanical durability of round clinched steel/aluminium joints. International Journal of Mechanical and Materials Engineering 2014 9:23.

\section{Submit your manuscript to a SpringerOpen ${ }^{\odot}$ journal and benefit from:}

- Convenient online submission

- Rigorous peer review

- Immediate publication on acceptance

- Open access: articles freely available online

- High visibility within the field

- Retaining the copyright to your article

Submit your next manuscript at $>$ springeropen.com 